\title{
Alpha and Theta Transcranial Alternating Current Stimulation Over the Right Dorsolateral Prefrontal Cortex Modulates Vigilance Performance, but Only When Arousal Levels Are Non-optimal
}

\author{
Víctor Martínez-Pérez \\ University of Murcia \\ Miriam Tortajada \\ University of Murcia \\ Lucía B. Palmero \\ University of Murcia \\ Guillermo Campoy \\ University of Murcia \\ Luis J. Fuentes ( $\square$ lfuentes@um.es ) \\ University of Murcia
}

\section{Research Article}

Keywords: Current theoretical accounts, Alpha and theta transcranial, right dorsolateral, vigilance performance, arousal levels

Posted Date: July 30th, 2021

DOl: https://doi.org/10.21203/rs.3.rs-753599/v1

License: (c) (i) This work is licensed under a Creative Commons Attribution 4.0 International License.

Read Full License 


\section{Abstract}

\section{Background}

Current theoretical accounts on the oscillatory nature of sustained attention predict that entrainment via transcranial alternating current stimulation (tACS) at alpha and theta frequencies on the frontoparietal network could prevent the drops in vigilance across time-on-task. Nonetheless, most previous studies have neglected both the fact that vigilance comprises two dissociable components (i.e. arousal and executive vigilance) and the potential role of differences in arousal baseline.

\section{Method}

We examined the effects of theta- and alpha-tACS over the right dorsolateral prefrontal cortex on both components of vigilance and on participants that differed in arousal baseline according to their chronotype and the time of testing. Intermediate-types performed the vigilance tasks when their arousal baseline was at the optimal level, whereas evening-types performed the vigilance tasks when their arousal baseline was at non-optimal levels.

\section{Results}

Both theta- and alpha-tACS improved arousal vigilance, whereas alpha-tACS, but not theta-tACS, improved accuracy and attenuated the typical vigilance decrement in the executive vigilance task. Importantly, these stimulation effects were only found when arousal baseline was low (i.e., with eveningtypes performing the tasks at their non-optimal time of day).

\section{Conclusion}

The results support the multicomponent view of vigilance, the relevance of heeding individual differences in arousal baseline, and the role of alpha oscillations as a long-range cortical scale synchronization mechanism that compensates the decrements in performance as a function of time-on-task by exerting and maintaining cognitive control attributed to activation of the frontoparietal network.

\section{Introduction}

A variety of jobs require workers to be vigilant for rather extended periods of time. This is the case of taxi drivers when travelling long distances, pilots in long-haul flights, or surgeons when performing an operation, among many other examples. The ability to maintain attention also plays a fundamental role in academic and clinical settings. Typically developing children usually maintain concentration on teachers' lectures to understand and retain large amount of information, an ability that children diagnosed with attention deficit hyperactivity disorder or autism spectrum disorder find extremely challenging. Detecting infrequent events is another type of activity that also requires maintaining attention throughout the activity, as occurs, for instance, when traffic controllers check for violations of traffic rules under congestion conditions or when teachers check for spelling mistakes in pupils' reports. A 
common phenomenon when the aforementioned real-life activities are simulated in laboratory is the socalled vigilance decrement, which entails an impairment in performance (i.e., longer RTs and/or lower accuracy) with time-on-task [1-3]. However, the concept of vigilance is not unitary, and several forms of sustained attention may be involved in different vigilance tasks. Also, of special relevance in social, educational, and clinical settings is whether such vigilance decrements can be counteracted, and whether the effectivity of improvement procedures will depend on individual differences in arousal baseline. Here we set out to address these important issues given the relevance that vigilant attention has in many spheres of our life.

\section{Components of vigilant attention}

According to Posner's neurocognitive approach to attention, the alerting network is involved in both transiently preparing the individual to perceive and/or respond to a forthcoming target, which is referred to as the phasic component of the network, and in achieving and maintaining an optimal level of activation for longer periods of time (sustained attention), which is referred to as the tonic component of the network $[4,5]$. A right lateralized cortical network including the anterior cingulate cortex, the dorsolateral prefrontal cortex (DLPFC) and the right inferior parietal lobe [6] (see e.g. Ref. [7], for a review) has been involved in the tonic component, also referred to as vigilance. Accordingly, time-related drops in vigilance have been shown to correlate with right frontoparietal deactivation [8]. Likewise,

electrophysiological studies have further shown that time-on-task variations in vigilance have been linked to the amplitude of theta and alpha cortical oscillations in the frontoparietal systems [9].

Further dissociations in the tonic component of the alerting network have been recently observed when different vigilance tasks are to be performed [10]. When the task is rather monotonous and tedious, with scarce requirements of cognitive or motoric demands, a kind of arousal vigilance mechanism is activated to maintain an optimal arousal level, allowing faster responses to stimuli of the environment. A prototypical task of this kind is the psychomotor vigilance task (PVT) [11], where participants are told to respond as quickly as possible once a randomly presented target (e.g., a colored point) is detected. However, when the task makes strong demands of cognitive processes, such as in resolving conflict, flexibly switching between tasks, or withholding respond to infrequent targets, a kind of executive vigilance is then recruited [10]. A prototypical task of this kind is the sustained attention to response task (SART) [12], where participants are told to respond to a succession of stimuli but inhibit the response just when an infrequent and randomly occurring target (e.g., a specific digit) is presented.

Beside both time-on-task and type of task, vigilance is affected by two important factors that determine how efficiently people sustain attention in vigilance tasks, the individual differences in circadian rhythms (e.g., chronotype [13]) and the oscillatory nature of attention (e.g., cortical oscillations in the frontoparietal network [9]).

\section{Chronotype in vigilance tasks}


In synchrony with external time, circadian rhythms, our endogenous biological clock, determine our physiological and behavioral processes. Levels of arousal tend to stabilize along daytime, when the circadian rhythm system compensates the sleep-regulation homeostatic system, which accumulates pression to sleep depending on the time an individual spent awake [14]. However, as people undergo shifts in circadian phase, they may differ in their peak times where their levels of arousal are high [14-16] leading to different circadian phenotypes that are usually classified as chronotypes. Thus, chronotype refers to the time of day preferred by individuals to perform their daily life activities and to sleep, which may result in morning-, evening- or intermediate-types. Morning-types reach their optimal functioning early in the morning, evening-types reach it late in the evening, and intermediate-types (the more frequent chronotype) are characterized by not having a pronounced circadian preference [14]. Although there are some physiological indices to determine people's preferences for performing daily activities and sleeping, questionnaires have proven to be suitable for classify individuals according to their chronotype [17].

Martínez-Pérez et al. [13] showed that evening-types performed better at their optimal time of day compared with their non-optimal time of day in both a task involving arousal vigilance (the PVT) and a conflict task that required executive vigilance (the flanker task). Morning-types, however, only showed this kind of synchrony effect in the PVT, and this effect was, moreover, smaller in comparison with that found with evening-types. These findings suggest that fluctuations in vigilance are more apparent in eveningtypes than in morning-types individuals (see also [18]).

\section{Performance modulation in vigilance tasks}

Some electrophysiological studies have shown that time-on-task variations in sustained attention can be modulated by non-invasive brain stimulation (NIBS) [19-28]. A first set of studies used transcranial direct current stimulation (tDCS) protocols [19, 23-27]. Luna et al. [23], for example, found that $1.5 \mathrm{~mA}$ of anodal high-definition tDCS over either the posterior parietal cortex or the DLDFC mitigated the executive vigilance decrement across time-on-task, whereas stimulation did not modulate the arousal component of vigilance. A second set of studies used transcranial alternating current stimulation (tACS) protocols. This technique has been proven to boost cognitive performance by enhancing the transfer of information among anatomically and functionally connected brain areas, which improve cognitive processes when the current is applied at specific oscillatory frequencies that concur with the endogenous regional synchronization involved in such cognitive functions. Both theta and alpha activity within the frontoparietal control network have been associated with either an increase or a decrease of cognitive control that are thought to be crucial for vigilance [29-40]. Congruently, and in accordance with the oscillatory models of sustained attention $[20,28]$, previous tACS studies on sustained attention stimulated at these two frequencies. For instance, Clayton et al. [20] found that $2.0 \mathrm{~mA}$ of alpha-tACS (10 $\mathrm{Hz}$ ) over occipitoparietal cortex prevented deterioration in two different vigilance tasks. The authors concluded that alpha oscillations promote top-down control processes and vigilance stability. Rostami et al. [28] tested the effects of $1 \mathrm{~mA}$ theta-tACS $(6 \mathrm{~Hz})$ over the medial prefrontal cortex and found pre-post stimulation differences in both frontal-midline theta power and performance on a sustained attention task. To our knowledge, however, none of these previous studies have simultaneously considered the 
three key factors regarding sustained attention in vigilance tasks: the multicomponent nature of vigilance, the potential role of individual differences in arousal level at baseline, and the oscillatory nature of sustained attention. Luna et al. [23] took into account the distinction between arousal and executive components of vigilance [10], but they did not heed neither the individual differences in arousal levels at baseline nor the oscillatory nature of sustained attention. Clayton et al. [20] and Rostami et al. [28], for their part, considered the oscillatory nature of sustained attention, but they did not heed neither the different components of vigilance, nor individual differences in arousal baseline.

Recent studies have highlighted the relevance of individual differences when assessing different methods of cognitive enhancing [21] (see Refs. [41, 42], for recent reviews). In some cases, maximal effectivity is expected when people's cortical excitability is below an optimal value according to an inverted U-shaped distribution, whereas either no effect or adverse effects are expected when the level of cortical excitability is at either optimal levels or above. Thus, it seems that NIBS effects could be modulated by pre-existing cortical excitation and inhibition baseline depending on factors such as age, hormonal fluctuations, neurotransmitter levels, and importantly, circadian influences [41]. The closer an individual is to its theoretical optimal arousal level, the lower the gain from stimulation is expected to be. In contrast, at suboptimal arousal levels gains may become greater [42]. Although previous studies have not purposely controlled for the influence of these individual differences at baseline, reliable tDCS effects have been observed when participants were at a non-optimal level of arousal due to sleep deprivation [24, 25], aging [19], or some pathological conditions [43].

\section{The present study}

In two experiments, we set out to modulate vigilance performance in two tasks, one thought to tap arousal vigilance (the PVT) and other thought to tap executive vigilance (the SART). We investigated whether the two types of vigilance are causally related to frontoparietal theta and alpha rhythms by applying HD-tACS to the DLPFC, in line with previous related studies that targeted that region of the frontoparietal network for stimulation (e.g., [23, 24]). Differences in arousal levels were addressed by considering participants' chronotype and time of testing. We tested intermediate-types at the time of day when their arousal level was deemed to be optimal (Experiment 1) and evening-types at the time of day when their arousal level was expected to be low (Experiment 2). We hypothesized that evening-types would benefit more than intermediate-types from applying theta/alpha HD-tACS when performing the vigilance tasks, due to the former having lower baseline level of arousal than the latter at the time of testing.

\section{Methods}

\section{Participants}

Two hundred and fifty undergraduates from the University of Murcia completed (online) the reduced version of the Horne and Östberg's Morningness-Eveningness Questionnaire (rMEQ) developed by Adan and Almirall [44]. The rMEQ consisted of five items, with total scores ranging from 4 (definitively evening- 
types) to 25 (definitively morning-types). From this initial sample, undergraduates classified as intermediate-types (rMEQ scores from 12 to 16) and evening-types (rMEQ scores from 4 to 11) were invited to participate in Experiments 1 and 2, respectively. Seventy-three intermediate-types (57 females; $M$ age $=20.30, S D=2.59 ; M r M E Q$ score $=15.34)$ agreed to participate in Experiment 1 and 29 eveningtypes (27 females; $M$ age $=19.62, S D=2.06$; M rMEQ score $=9.17$ ) agreed to participate in Experiment 2 . The difference between experiments in the number of participants was unintended and resulted from the lower number of evening-types in the initial sample (and in the general population).

All participants reported normal or corrected-to-normal vision and no chronic medical conditions. They gave written informed consent and received course credits for their participation. This study was approved by the Ethics Committee of the University of Murcia and was conducted conformed with the ethical standards laid down in the 1964 Declaration of Helsinki.

\section{General procedure}

Experiment 1 and 2 were ran in parallel as intermediate- and evening-types participants were recruited. All participants were asked not to drink coffee or other stimulants for at least two hours prior to the tests. Participants from Experiment 1 (intermediate-types) came to the laboratory at 10:00 AM, 11:30 AM or 1:00 PM, times of the day when arousal is supposed to be at an optimal level for non-extreme chronotypes. Participants from Experiment 2 (evening-types) were cited at 8:00 AM, when their arousal level was expected to be low. We chose a parallel instead of a crossover design to avoid the potential learning effects that could conceal tACS effects [22]. Thus, participants came to the laboratory only on one occasion. Once in the laboratory, they were interviewed about their stimulant intake (none of them reported consumption). Next, they were randomly assigned to one of the three tACS stimulation conditions (sham, alpha, and theta), with the restriction that the number of participants per condition was equalized every three participants. The final number of participants in the sham, alpha and theta conditions were, respectively, 24, 24, and 25 in Experiment 1; and 9, 10, and 10 in Experiment 2. Then, while receiving 25 minutes online stimulation, they performed the PVT for 10 minutes and, right after, the SART for about 18 minutes. Finally, participants were asked to rate their sensations related to stimulation (itching, pain, heat, etc.) from 0 to 10 (stimulation groups did not differ in self-report sensations, $F_{s}<1$ in the two experiments). In total, participants remained in the laboratory for approximately 45 minutes.

\section{Behavioral tasks}

The tasks were controlled by software written in E Prime [45]. Participants were seated at a viewing distance of approximately $60 \mathrm{~cm}$ from the center of a 22-inch computer screen (resolution: 1920 by 1080 pixels) and responded using a Chronos ${ }^{\circledR}$ response box (Psychology Software Tools).

In the PVT, participants had to press a button as fast as possible when a red circle appeared on the screen. Each trial began with a random blank interval between 2 and $10 \mathrm{~s}$. Then a red circle of 50 pixels in diameter popped up in the center of the screen and participants had to press a button. Then, the screen went blank and a new trial began. 
In the SART, digits from 1 to 9 appeared in the center of the screen and participants had to press a button in response to all digit except the digit 3. Digits appeared in different font sizes $(18,27,36,45$, or 54 points) for $250 \mathrm{~ms}$, followed by an 800-ms mask (a circle with a cross inside) and a 100-ms blank screen (presentation rate: one digit every $1150 \mathrm{~ms}$ ). Digits were selected at random with the restriction that each digit appeared once every nine trials and that the same digit did not appear in two consecutive trials. Participants completed 900 trials preceded by 18 practice trials. They were encouraged to respond quickly but making as few errors as possible.

\section{Stimulation}

Transcranial stimulation was delivered through a StarStim ${ }^{\circledR}$ wireless neurostimulator system (Neuroelectrics, Barcelona, Spain) connected to circular sponge-based electrodes $\left(8 \mathrm{~cm}^{2}\right)$, saturated with saline solutions to keep impedances below $10 \mathrm{k} \Omega$. The target area of stimulation was the right DLPFC, located in F4 based on the 10-20 system (Fig. 1). Three return electrodes were placed in a triangular scheme (T8, Cz, and Fp1), each of them with a 33\% of current return (Fig. 2). Both experimenters and participants were blind to the stimulation conditions. During alpha- and theta-tACS, $1.5 \mathrm{~mA}$ intensity stimulation was applied at $10 \mathrm{~Hz}$ and $6 \mathrm{~Hz}$, respectively, right from the beginning of the PVT (including 30 $\mathrm{s}$ of ramp-up/down) and for $25 \mathrm{~min}$. Sham-tACS stimulation was applied at $10 \mathrm{~Hz}$ ( $1.5 \mathrm{~mA}$ intensity) only at ramp periods to emulate the skin tingling sensation. It has been proven that this type of high-definition montages improve the focality of stimulation [46-48].

\section{Statistical analyses}

We analyzed the reaction times (RTs) on the PVT, the accuracy on the no-go trials of the SART (i.e., the proportion of no-go trials in which participants withheld their response), and the RTs on go trials of the SART. To detect possible changes in performance throughout the course of the tasks, we divided the PVT into five two-minute blocks (with between 12 and 23 trials per block, $M=18.69$ ) and the SART into five blocks of 180 trials (160 go and 20 no-go). RTs were log-transformed to reduce the skewing in their distribution, and log-RTs beyond four times the semi-interquartile range from the median $(0.41 \%$ and $0.58 \%$ of the data in the PVT and the SART, respectively) were considered outliers and removed. The results were obtained by a series of $3 \times 5$ mixed analyses of variance (ANOVA), with stimulation (alpha, theta, sham) as the between-participants factor and block (1-5) as the within-participants factor. The Greenhouse-Geisser sphericity correction was applied when necessary. Subsequent post-hoc tests used the Bonferroni method to correct for multiple comparisons. Additional independent $t$-tests were also conducted. We adopted a significance level of .05 for all analyses, which were performed with JASP 0.14 [49].

\section{Results}

Figure 2 depicts the mean RT and accuracy across experiments and conditions. Table 1 shows the statistics of the ANOVAs performed on the three variables considered (RT on the PVT, accuracy on the SART, and RT on the SART) for each experiment. 
Table 1

Statistical results in Experiments 1 and 2 for the 3 (stimulation, S) $\times 5$ (block, B) mixed ANOVAs on the RTs in the PVT, accuracy in the no-go trials of the SART, and RTs in the go trials of the SART.

\begin{tabular}{|c|c|c|c|c|c|c|c|c|c|}
\hline \multirow[t]{2}{*}{ Variable } & \multirow[t]{2}{*}{ Effect } & \multicolumn{4}{|c|}{ Experiment 1} & \multicolumn{4}{|c|}{ Experiment 2} \\
\hline & & $\mathrm{F}$ & $d f$ & $\mathrm{p}$ & $\eta_{p}^{2}$ & $\mathrm{~F}$ & $d f$ & $\mathrm{p}$ & $\eta_{p}^{2}$ \\
\hline \multirow[t]{3}{*}{ PVT, RT } & $S$ & 2.17 & 2,70 & .122 & .058 & 4.18 & 2,26 & .027 & .243 \\
\hline & B & 14.40 & $2.4,167.8$ & $<.001$ & .171 & 5.89 & $\begin{array}{l}2.3 \\
60.5\end{array}$ & .003 & .184 \\
\hline & $S \times B$ & 0.25 & $4.8,167.8$ & .933 & .007 & 1.48 & $\begin{array}{l}4.7 \\
60.5\end{array}$ & .212 & .102 \\
\hline \multirow{3}{*}{$\begin{array}{l}\text { SART, } \\
\text { accuracy }\end{array}$} & $S$ & 0.28 & 2,70 & .757 & .008 & 6.68 & 2,26 & .005 & .340 \\
\hline & B & 8.72 & $\begin{array}{l}3.38 \\
236.4\end{array}$ & $<.001$ & .111 & 8.92 & 4,104 & $<.001$ & .255 \\
\hline & $S \times B$ & 0.77 & $6.8,236.4$ & .612 & .021 & 1.39 & 8,104 & .211 & .096 \\
\hline \multirow[t]{3}{*}{ SART, RT } & S & 1.09 & 2,70 & .341 & .030 & 5.44 & 2,26 & .011 & .295 \\
\hline & B & 3.12 & $3.6,241.5$ & .021 & .043 & 6.20 & 4,104 & $<.001$ & .193 \\
\hline & $S \times B$ & 0.57 & $6.9,241.5$ & .777 & .016 & 1.62 & 8,104 & .127 & .111 \\
\hline
\end{tabular}

Note: $\mathrm{S}$ = stimulation, $\mathrm{B}$ = block; the Greenhouse-Geisser sphericity correction was applied when necessary.

ANOVAs for Experiment 1 yielded statistically significant main effects of block, showing that performance in the two tasks declined as these tasks progressed. Post-hoc tests for the PVT revealed faster RTs in the first two blocks than in the last two blocks and faster RTs in the third block than in the last one, ts > 3.9. On the other hand, post-hoc tests for the SART revealed better accuracy in the first block than in the other four blocks, $t s>3.0$, and slower RTs in the first block than in the last one, $t=3.1$ (note that performance decline in the SART is revealed by faster rather than slower RTs). Likewise, ANOVAs for the Experiment 2 yielded main effects of block, showing performance deterioration throughout the tasks. Post-hoc tests revealed faster RTs in the first two blocks of the PVT than in the last two blocks, $t s>3.2$, better accuracy in the first block of the SART than in the other four blocks, $t s>3.0$, and slower RTs in the first block of the SART than in the other four blocks, ts > 3.1. Importantly, in contrast to Experiment 1 , analyses for Experiment 2 also yielded statistically significant main effects of stimulation. For the PVT, post-hoc tests comparing the sham condition to each of the other stimulation conditions revealed slower RTs in the sham condition than in both the alpha condition, $t=2.60$, Cohen's $d=0.484$, and the theta condition, $t=2.44$, Cohen's $d=0.453$. On the other hand, post-hoc tests for the SART revealed better accuracy in the alpha condition of the SART than in the sham condition, $t=3.40$, Cohen's $d=0.630$, and slower RTs in the alpha condition than in the sham condition, $t=3.01$, Cohen's $d=0.559$; the theta and the sham conditions did not differ, both $t s<1$. In Experiment 2, therefore, participants in the alpha condition 
performed better that the control (sham) group in both the PVT and the SART, whereas participants in the theta condition performed better that the control group in the PVT.

Regarding Experiment 2, an inspection of Fig. 2 (right panels) suggests that the better performance in the alpha condition resulted, at least in part, from a smaller performance decrement throughout the tasks, especially in the SART. To further analyze the decrement in performance with time-on-task in Experiment 2 , we compared the first and the last block across stimulation conditions by independent $t$-tests (Table 2). None of these comparisons reached the statistical significance level for the alpha condition (Cohen's $d s<$ 0.3 ), whereas, in the theta condition, there were significant performance decrements in all cases (Cohen's $d s>1$ ). In the sham condition, there were performance decrements in the SART, but the comparison for the PVT did not reach the statistical significance level.

Table 2

Comparisons between first and last blocks of trials in the dependent variables (RTs and accuracy) for both the PVT and the SART tasks.

\begin{tabular}{|llllll|}
\hline Stimulation & Variable & $\mathbf{t}$ & $\mathbf{d f}$ & $\mathbf{p}$ & Cohen's d \\
\hline Alpha & PVT, RT & 0.70 & 9 & .503 & 0.221 \\
& SART, accuracy & 0.87 & 9 & .405 & 0.276 \\
\hline \multirow{3}{*}{ Theta } & SART, RT & 0.58 & 9 & .580 & 0.182 \\
& PVT, RT & 3.19 & 9 & .011 & 1.009 \\
\hline \multirow{3}{*}{ Sham } & SART, accuracy & 3.82 & 9 & .004 & 1.209 \\
\hline \multirow{3}{*}{} & SART, RT & 3.57 & 9 & .006 & 1.128 \\
\hline & PVT, RT & 1.22 & 8 & .257 & 0.407 \\
\hline & SART, accuracy & 3.01 & 8 & .017 & 1.004 \\
\hline
\end{tabular}

Note: A greater effect size (Cohen's d) represents a larger decrease in performance.

\section{Discussion}

Sustained attention is crucial in many of our daily life activities, activities that have been simulated in the laboratory using vigilance tasks. However, the concept of vigilance is not unitary, and several components can be dissociated at both the behavioral and neural level [10,23] (see [7], for a review). In the present experiments, we have observed that both an arousal component, mainly involved in tedious and monotonous tasks (e.g., the PVT), and an executive component, mainly involved in vigilance tasks that require inhibitory control (e.g., the SART), can be differently modulated by non-invasive brain stimulation methods, as a function of individual differences in arousal baseline based on chronotype. When participants carry out tasks that require sustained attention in times of the day that, according to their chronotypes, match with their non-optimal level of arousal, performance is seriously affected in 
comparison to when they carry out the tasks in their optimal time of day [13]. Arousal baseline may be linked to the biological aspects of the circadian rhythms that, in interaction with the preference of individuals about when to perform their day life activities, generate variable levels of activation that affect their performance. Given that circadian influences affect cortical excitability [50-52], we suggest that pre-existing excitation/inhibition baseline levels may determine whether brain stimulation will or will not have any effect on performance [41]. Accordingly, only evening-types, who performed the vigilance tasks at the non-optimal level of arousal (early in the morning) benefited from HD-tACS. These results agree with previous studies showing that different subgroups of participants with different baseline levels of cortical activation responded differentially to neuromodulation [21, 53-55].

People not only differ in arousal levels along the day, but also in their ability to sustain attention for extended periods of time. With time-on-task, participants usually show a progressive decrement in performance due to a decline in arousal levels that would affect their ability to sustain attention throughout the task. Due to the monotonous nature of some repetitive tasks that make scarce requirements of cognitive resources, it is expected that participants diminish their interest and lose the focus on the task. In previous research, we have observed that evening-types participants showed the synchrony effect, that is, they produced longer RTs when they performed the monotonous task (PVT) at their non-optimal time of day compared with when they performed the task at the optimal time of day [13]. Importantly, when the RT distribution was computed, the synchrony effect became larger at the slower end of the distribution, that is, when extreme fluctuations of attention emerged at the non-optimal time of day. These results suggest that sustained attention required in monotonous tasks fluctuates, mainly when the task must be carried out under low arousal conditions.

Boosting general arousal through non-invasive brain stimulation, irrespective of whether electrical current oscillated at theta- or alpha-frequencies, has proved also as an appropriate technique to overcome the decrement in performance that it is usually observed in arousal vigilance tasks such as the PVT. Here we show that entrainment at those oscillations improved performance but only when the arousal component of vigilance was at non-optimal levels, that is, when evening-types participants carried out the task early in the morning. One plausible explanation is that for the arousal component, brain stimulation has a general booster effect that shortens RTs, which would not be dependent on the concrete oscillation that has been entrained. A similar effect is even found when tDCS is applied over the prefrontal cortex under conditions of sleep deprivation [24]. Thus, it is activation of the frontoparietal network by NIBS techniques what seems to cause an increment in arousal vigilance, irrespective of the oscillatory nature of the brain stimulation protocol to be used.

In contrast, when the task required strong demands of cognitive control, for instance, by asking to withhold responding just to infrequent targets (no-go trials of the SART), only stimulation at alpha oscillations improved executive vigilance performance, and also attenuated vigilance decrements in comparison to both theta oscillations and sham. Oscillations at the alpha frequency would have shown differentiated roles in the current study. Alpha oscillations have been thought to exert an overall inhibitory effect on cortical processing, but they also contribute to top-down inhibitory control mechanisms 
affecting task-irrelevant processes (see Ref. [9], for review) that involve the prefrontal regions of the frontoparietal network [56]. In this line, such top-down modulation has been associated with frontalparietal coherence in the alpha band [57]. In the SART, alpha oscillations may have caused suppression of irrelevant stimuli (non-target digits) leading to increased RTs in go trials. Alpha oscillations have also been found to facilitate attentional stability $[9,20]$. Our findings fit well with such contention. By guiding attentional resources to the relevant stimulus (infrequent target digit), accuracy in withholding responses in no-go trials increased, fostering a high level of performance. Importantly, the typical vigilance decrement observed with time-on-task was also attenuated. These effects on no-go trials accuracy may reflect a selective role of long-range synchrony effects of alpha oscillations in the activity of several brain areas that correspond with the frontoparietal network, and hence with the cognitive operations supported by this network [36]. One relevant operation is concerned with phasic aspects of cognitive control, which can be triggered exogenously, for instance when an error has been committed [58], and, of special relevance for the purpose of the present study, by salient target stimuli in a bottom-up manner. Bottom-up activation of cognitive control here may have been triggered by the sudden appearance of the infrequent target digit, which would activate the initiation of inhibitory control to avoid responding to it. Other relevant operation is concerned with the top-down maintenance (working memory) of task requirements, mainly involving the DLPFC $[59,60]$. Top-down maintenance of information in working memory is required here when participants are given the instructions of responding to irrelevant frequent non-target stimuli and withholding responses just to the relevant infrequent digit 3.

These results support the model proposed by Clayton et al. [9] by further providing it with causal evidence. This model predicts that performance in tasks that require sustained attention will be improved by entraining endogenous alpha and theta oscillations via tACS in frontal areas. Mechanistically, entrainment at both theta and alpha frequencies would promote changes in excitability of the frontoparietal circuit involved in vigilant attention [7]. The present results suggest that a more compelling model should heed the different components involved in vigilance tasks as well as the individual differences in arousal at baseline.

A remaining issue concerns the failure to observe any modulation of executive vigilance performance by applying theta-tACS. Fluctuations in cognitive control have been associated to power of theta band oscillations (e.g. [61]), mainly in conflict tasks [9]. Thus, an effect of such slow oscillations should be expected mainly in the maintenance of task requirements in working memory, an operation that requires cognitive control. One possibility is that the typical theta oscillations at the local midfrontal region under full alertness condition, is no longer noticeable when alertness levels decrease, either because tasks are to be perform at non-optimal times of day, or because participants become drowsy [62]. Under such conditions, people are less capable of implementing cognitive control required by attentional demanding tasks. One way of counteracting the negative effects of low levels of arousal on maintaining cognitive control with time-on-task is to activate a reconfiguration of the cognitive control system via long-range cortical scale synchronization mechanisms between brain regions. The frequency band of such synchronization might depend on the kind of cognitive control required by the task at hand. In case of conflict tasks, theta band oscillations seem to be the more appropriate $[9,62]$. In case of tasks such as 
the SART, our current results suggest alpha band oscillations as the most appropriate. In any case, the result is that performance in such tasks is kept at the level of what is expected under conditions of full alertness. Thus, we claim that a main role of alpha oscillations at the long-range is to compensate the decrements in performance as a function of time-on-task by exerting and maintaining cognitive control attributed to the frontoparietal network. Future work using EEG and NIBS protocols will help determine how alpha-frequency oscillations communicate with the rest of the brain when people perform executive vigilance tasks under low arousal conditions.

\section{Conclusions}

To conclude, many daily activities require sustained attention for extended periods of time. For instance, in labor contexts, some jobs require working on nocturnal turns under sleep deprivation conditions, increasing fatigue and hence the risk of human error $[63,64]$. In education contexts, where classes are usually scheduled in the morning, students whose high level of arousal and cortical excitability occur in the morning hours (morning-types chronotype) would be in more favorable conditions than students with evening-types chronotype whose optimal level of arousal and cortical excitability occur in the evening, and vice versa when classes are scheduled in the evening [65].

Recent studies have shown that non-invasive brain stimulation methods pave the way to become an appropriate countermeasure to overcome the deleterious effect of vigilance decrements well beyond other common methods frequently used in different fields such as caffeine intake [24] or the use of nootropic drugs such as modafinil [66].

In the present study we went further to explore the benefits of brain stimulation in vigilance tasks by overcoming some shortcomings of previous studies. We dissociated two components of vigilance, one arousal component characteristic of monotonous tasks such as the PVT, and an executive component characteristic of cognitive demanding tasks such as the SART. We heeded individual differences in arousal baseline by testing participants at the optimal or non-optimal time of day according to their chronotype. Finally, we observed that the effect of brain stimulation on both tasks very much depended on stimulating either theta or alpha rhythms by using high-definition transcranial alternating current stimulation (HD-tACS), according to the fluctuation nature of sustained attention. Our results showed that tACS stimulation, irrespective of applying concrete oscillation rhythms, boosted general cortical activation enhancing performance in the arousal component of vigilance. However, only stimulation at alpha frequency improved performance in the executive component of vigilance as well as attenuated the typical vigilance decrement usually observed with time-on-task. These results were observed just in evening-types participants tested when their levels of arousal were non-optimal, but not in intermediatetypes participants tested when their level of arousal were optimal, highlighting the relevance of taking into account individual differences in pre-existing excitation/inhibition baseline levels in assessing cognitive enhancement.

\section{Declarations}




\section{Author contributions}

VMP: Conceptualization, Methodology, Formal analysis, Investigation, Writing-Original draft preparation.

MT: Conceptualization, Investigation, Writing- Original draft preparation. LBP: Conceptualization, Investigation, Writing- Original draft preparation. GC: Conceptualization, Methodology, Formal analysis, Writing-Original draft preparation, Writing-Reviewing and Editing. LJF: Conceptualization, Methodology, Writing-Original draft preparation, Writing-Reviewing and Editing, Funding acquisition.

\section{References}

1. Davies DR, Parasuraman R. The psychology of vigilance. London: Academic Press; 1982.

2. Helton WS, Warm JS. Signal salience and the mindlessness theory of vigilance. Acta Psychologica 2008;129:18-25. https://doi.org/10.1016/j.actpsy.2008.04.002.

3. Mackworth NH. The Breakdown of Vigilance during Prolonged Visual Search. Quarterly Journal of Experimental Psychology 1948;1:6-21. https://doi.org/10.1080/17470214808416738.

4. Petersen SE, Posner MI. The Attention System of the Human Brain: 20 Years After. Annu Rev Neurosci 2012;35:73-89. https://doi.org/10.1146/annurev-neuro-062111-150525.

5. Posner MI, Petersen SE. The attention system of the human brain. Annual Review of Neuroscience 1990;13:25-42. https://doi.org/10.1146/annurev.ne.13.030190.000325.

6. Sturm W, Willmes K. On the functional neuroanatomy of intrinsic and phasic alertness. Neuroimage 2001;14:S76-84.

7. Robertson IH, O'Connell R. Vigilant attention. Attention and Time, Oxford: Oxford University Press; 2010. https://doi.org/10.1093/acprof:oso/9780199563456.003.0006.

8. Langner R, Eickhoff SB. Sustaining attention to simple tasks: A meta-analytic review of the neural mechanisms of vigilant attention. Psychological Bulletin 2013;139:870-900. https://doi.org/10.1037/a0030694.

9. Clayton MS, Yeung N, Cohen Kadosh R. The roles of cortical oscillations in sustained attention. Trends in Cognitive Sciences 2015;19:188-95. https://doi.org/10.1016/j.tics.2015.02.004.

10. Luna FG, Marino J, Roca J, Lupiáñez J. Executive and arousal vigilance decrement in the context of the attentional networks: The ANTI-Vea task. Journal of Neuroscience Methods 2018;306:77-87. https://doi.org/10.1016/j.jneumeth.2018.05.011.

11. Dinges DF, Powell JW. Microcomputer analyses of performance on a portable, simple visual RT task during sustained operations. Behavior Research Methods, Instruments, \& Computers 1985;17:652-5.

12. Robertson IH, Manly T, Andrade J, Baddeley BT, Yiend J. "Oops!": performance correlates of everyday attentional failures in traumatic brain injured and normal subjects. Neuropsychologia 1997;35:74758. https://doi.org/10.1016/s0028-3932(97)00015-8.

13. Martínez-Pérez V, Palmero LB, Campoy G, Fuentes LJ. The role of chronotype in the interaction between the alerting and the executive control networks. Sci Rep 2020;10:11901. 
https://doi.org/10.1038/s41598-020-68755-z.

14. Schmidt C, Collette F, Cajochen C, Peigneux P. A time to think: circadian rhythms in human cognition. Cognitive Neuropsychology 2007;24:755-89.

15. Levandovski R, Sasso E, Hidalgo MP. Chronotype: a review of the advances, limits and applicability of the main instruments used in the literature to assess human phenotype. Trends in Psychiatry and Psychotherapy 2013;35:3-11.

16. Valdez P, Ramírez C, García A. Circadian rhythms in cognitive performance: implications for neuropsychological assessment. ChronoPhysiology and Therapy 2012;2:81-92.

17. Horne JA, Östberg O. A self-assessment questionnaire to determine morningness-eveningness in human circadian rhythms. International Journal of Chronobiology 1976.

18. Lara T, Madrid JA, Correa Á. The vigilance decrement in executive function is attenuated when individual chronotypes perform at their optimal time of day. PloS One 2014;9:e88820.

19. Brosnan MB, Arvaneh M, Harty S, Maguire T, O'Connell R, Robertson IH, et al. Prefrontal modulation of visual processing and sustained attention in aging, a tDCS-EEG coregistration approach. Journal of Cognitive Neuroscience 2018;30:1630-45.

20. Clayton MS, Yeung N, Cohen Kadosh R. Electrical stimulation of alpha oscillations stabilizes performance on visual attention tasks. Journal of Experimental Psychology: General 2019;148:203.

21. Harty S, Cohen Kadosh R. Suboptimal engagement of high-level cortical regions predicts randomnoise-related gains in sustained attention. Psychological Science 2019;30:1318-32.

22. Jacoby N, Lavidor M. Null tDCS effects in a sustained attention task: the modulating role of learning. Frontiers in Psychology 2018;9:476.

23. Luna FG, Román-Caballero R, Barttfeld P, Lupiáñez J, Martín-Arévalo E. A High-Definition tDCS and EEG study on attention and vigilance: Brain stimulation mitigates the executive but not the arousal vigilance decrement. Neuropsychologia 2020;142:107447. https://doi.org/10.1016/j.neuropsychologia.2020.107447.

24. McIntire LK, McKinley RA, Goodyear C, Nelson J. A comparison of the effects of transcranial direct current stimulation and caffeine on vigilance and cognitive performance during extended wakefulness. Brain Stimulation 2014;7:499-507.

25. McIntire LK, McKinley RA, Nelson JM, Goodyear C. Transcranial direct current stimulation versus caffeine as a fatigue countermeasure. Brain Stimulation 2017;10:1070-8.

26. Nelson JT, McKinley RA, Golob EJ, Warm JS, Parasuraman R. Enhancing vigilance in operators with prefrontal cortex transcranial direct current stimulation (tDCS). Neuroimage 2014;85:909-17.

27. Roe JM, Nesheim M, Mathiesen NC, Moberget T, Alnæs D, Sneve MH. The effects of tDCS upon sustained visual attention are dependent on cognitive load. Neuropsychologia 2016;80:1-8.

28. Rostami R, Kazemi R, Mozaffarinejad F, Nasiri Z, Rostami M, L. Hadipour A, et al. $6 \mathrm{~Hz}$ transcranial alternating current stimulation of MPFC improves sustained attention and modulates alpha phase synchronization and power in dorsal attention network. Cognitive Neuroscience 2021;12:1-13. 
29. Ahveninen J, Huang S, Belliveau JW, Chang W-T, Hämäläinen M. Dynamic oscillatory processes governing cued orienting and allocation of auditory attention. Journal of Cognitive Neuroscience 2013;25:1926-43.

30. Barry RJ, Clarke AR, Johnstone SJ, Magee CA, Rushby JA. EEG differences between eyes-closed and eyes-open resting conditions. Clinical Neurophysiology 2007;118:2765-73.

31. Boksem MA, Meijman TF, Lorist MM. Effects of mental fatigue on attention: an ERP study. Cognitive Brain Research 2005;25:107-16.

32. Cavanagh JF, Frank MJ. Frontal theta as a mechanism for cognitive control. Trends in Cognitive Sciences 2014;18:414-21.

33. Cohen MX. A neural microcircuit for cognitive conflict detection and signaling. Trends in Neurosciences 2014;37:480-90.

34. Missonnier P, Deiber M-P, Gold G, Millet P, Pun MG-F, Fazio-Costa L, et al. Frontal theta event-related synchronization: comparison of directed attention and working memory load effects. Journal of Neural Transmission 2006;113:1477-86.

35. Oehrn CR, HansImayr S, Fell J, Deuker L, Kremers NA, Do Lam AT, et al. Neural communication patterns underlying conflict detection, resolution, and adaptation. Journal of Neuroscience 2014;34:10438-52.

36. Sadaghiani S, Scheeringa R, Lehongre K, Morillon B, Giraud A-L, d'Esposito M, et al. Alpha-band phase synchrony is related to activity in the fronto-parietal adaptive control network. Journal of Neuroscience 2012;32:14305-10.

37. Saalmann YB, Pinsk MA, Wang L, Li X, Kastner S. The pulvinar regulates information transmission between cortical areas based on attention demands. Science 2012;337:753-6.

38. Snyder AC, Foxe JJ. Anticipatory attentional suppression of visual features indexed by oscillatory alpha-band power increases: a high-density electrical mapping study. Journal of Neuroscience 2010;30:4024-32.

39. O'Connell RG, Dockree PM, Robertson IH, Bellgrove MA, Foxe JJ, Kelly SP. Uncovering the neural signature of lapsing attention: electrophysiological signals predict errors up to $20 \mathrm{~s}$ before they occur. Journal of Neuroscience 2009;29:8604-11.

40. Wascher E, Rasch B, Sänger J, Hoffmann S, Schneider D, Rinkenauer G, et al. Frontal theta activity reflects distinct aspects of mental fatigue. Biological Psychology 2014;96:57-65.

41. Krause B, Cohen Kadosh R. Not all brains are created equal: the relevance of individual differences in responsiveness to transcranial electrical stimulation. Frontiers in Systems Neuroscience 2014;8:25.

42. Colzato LS, Hommel B, Beste C. The Downsides of Cognitive Enhancement. The Neuroscientist 2020:1073858420945971.

43. Cachoeira CT, Leffa DT, Mittelstadt SD, Mendes LST, Brunoni AR, Pinto JV, et al. Positive effects of transcranial direct current stimulation in adult patients with attention-deficit/hyperactivity disorder $A$ pilot randomized controlled study. Psychiatry Research 2017;247:28-32. 
44. Adan A, Almirall $\mathrm{H}$. Horne \& Östberg morningness-eveningness questionnaire: A reduced scale. Personality and Individual Differences 1991;12:241-53.

45. Schneider W, Eschman A, Zuccolotto A. E-Prime reference guide. Psychology Software Tools, Incorporated; 2002.

46. Datta A, Bansal V, Diaz J, Patel J, Reato D, Bikson M. Gyri-precise head model of transcranial direct current stimulation: improved spatial focality using a ring electrode versus conventional rectangular pad. Brain Stimulation 2009;2:201-207. e1.

47. Kuo H-I, Bikson M, Datta A, Minhas P, Paulus W, Kuo M-F, et al. Comparing cortical plasticity induced by conventional and high-definition $4 \times 1$ ring tDCS: a neurophysiological study. Brain Stimulation 2013;6:644-8.

48. Martínez-Pérez V, Castillo A, Sánchez-Pérez N, Vivas AB, Campoy G, Fuentes LJ. Time course of the inhibitory tagging effect in ongoing emotional processing. A HD-tDCS study. Neuropsychologia 2019;135:107242.

49. JASP Team. JASP (Version 0.14.1)[Computer software]. 2020.

50. Alonzo A, Brassil J, Taylor JL, Martin D, Loo CK. Daily transcranial direct current stimulation (tDCS) leads to greater increases in cortical excitability than second daily transcranial direct current stimulation. Brain Stimulation 2012;5:208-13.

51. Huber R, Mäki H, Rosanova M, Casarotto S, Canali P, Casali AG, et al. Human cortical excitability increases with time awake. Cerebral Cortex 2013;23:1-7.

52. Lang N, Rothkegel H, Reiber H, Hasan A, Sueske E, Tergau F, et al. Circadian modulation of GABAmediated cortical inhibition. Cerebral Cortex 2011;21:2299-306.

53. London RE, Slagter HA. Effects of transcranial direct current stimulation over left dorsolateral pFC on the attentional blink depend on individual baseline performance. Journal of Cognitive Neuroscience 2015;27:2382-93.

54. López-Alonso V, Cheeran B, Río-Rodríguez D, Fernández-del-Olmo M. Inter-individual variability in response to non-invasive brain stimulation paradigms. Brain Stimulation 2014;7:372-80.

55. Santarnecchi E, Muller T, Rossi S, Sarkar A, Polizzotto NR, Rossi A, et al. Individual differences and specificity of prefrontal gamma frequency-tACS on fluid intelligence capabilities. Cortex 2016;75:3343.

56. Klimesch W. Alpha-band oscillations, attention, and controlled access to stored information. Trends in Cognitive Sciences 2012;16:606-17.

57. Zanto TP, Rubens MT, Thangavel A, Gazzaley A. Causal role of the prefrontal cortex in top-down modulation of visual processing and working memory. Nature Neuroscience 2011;14:656.

58. Dosenbach NU, Fair DA, Miezin FM, Cohen AL, Wenger KK, Dosenbach RA, et al. Distinct brain networks for adaptive and stable task control in humans. Proceedings of the National Academy of Sciences 2007;104:11073-8. 
59. D'Esposito M. From cognitive to neural models of working memory. Philosophical Transactions of the Royal Society B: Biological Sciences 2007;362:761-72.

60. MacDonald AW, Cohen JD, Stenger VA, Carter CS. Dissociating the role of the dorsolateral prefrontal and anterior cingulate cortex in cognitive control. Science 2000;288:1835-8.

61. Cohen MX, van Gaal S. Subthreshold muscle twitches dissociate oscillatory neural signatures of conflicts from errors. Neuroimage 2014;86:503-13.

62. Canales-Johnson A, Beerendonk L, Blain S, Kitaoka S, Ezquerro-Nassar A, Nuiten S, et al. Decreased alertness reconfigures cognitive control networks. Journal of Neuroscience 2020;40:7142-54.

63. Durmer JS, Dinges DF. Neurocognitive consequences of sleep deprivation. vol. 25, Copyright@ 2005 by Thieme Medical Publishers, Inc., 333 Seventh Avenue, New ...; 2005, p. 117-29.

64. Krueger GP. Sustained work, fatigue, sleep loss and performance: A review of the issues. Work \& Stress 1989;3:129-41.

65. Goldin AP, Sigman M, Braier G, Golombek DA, Leone MJ. Interplay of chronotype and school timing predicts school performance. Nature Human Behaviour 2020;4:387-96.

66. Baranski JV, Pigeau R, Dinich P, Jacobs I. Effects of modafinil on cognitive and meta-cognitive performance. Human Psychopharmacology: Clinical and Experimental 2004;19:323-32.

\section{Figures}
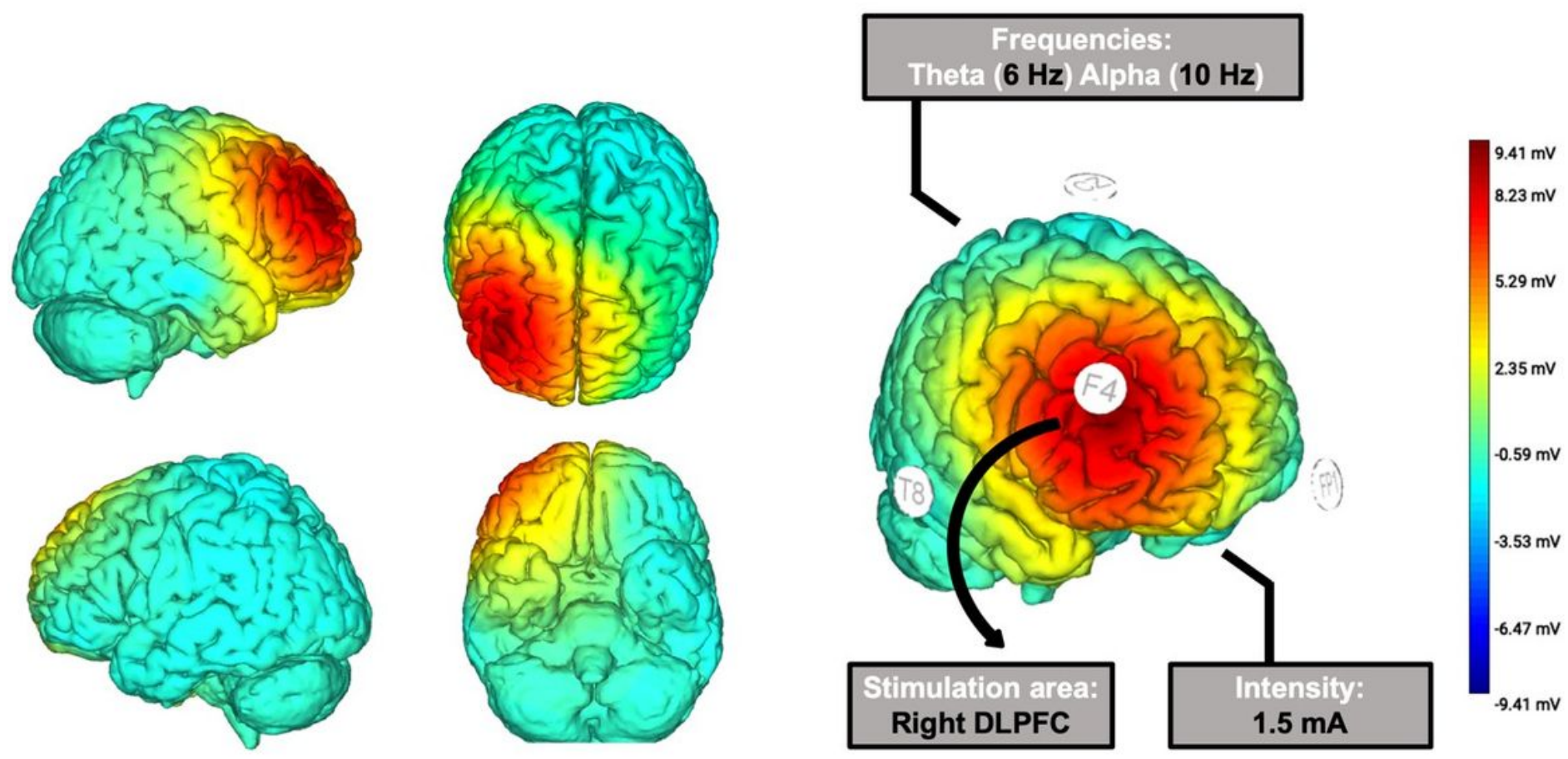

Figure 1 
An illustration of the HD-tACS montage and the simulation of the electric field generated according to the StimWeaver software (Neuroelectrics).

Exp. 1, PVT: RT

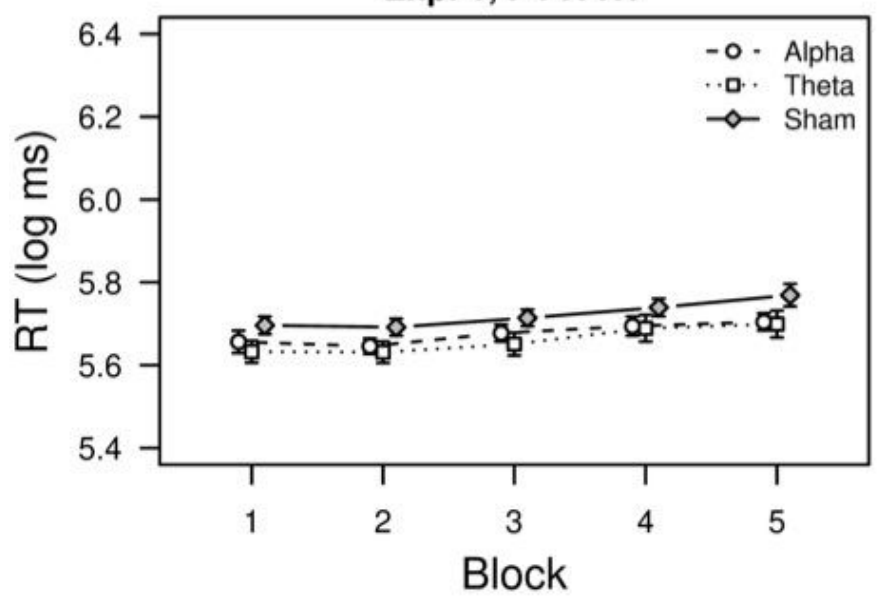

Exp. 1, SART: RT on go trials

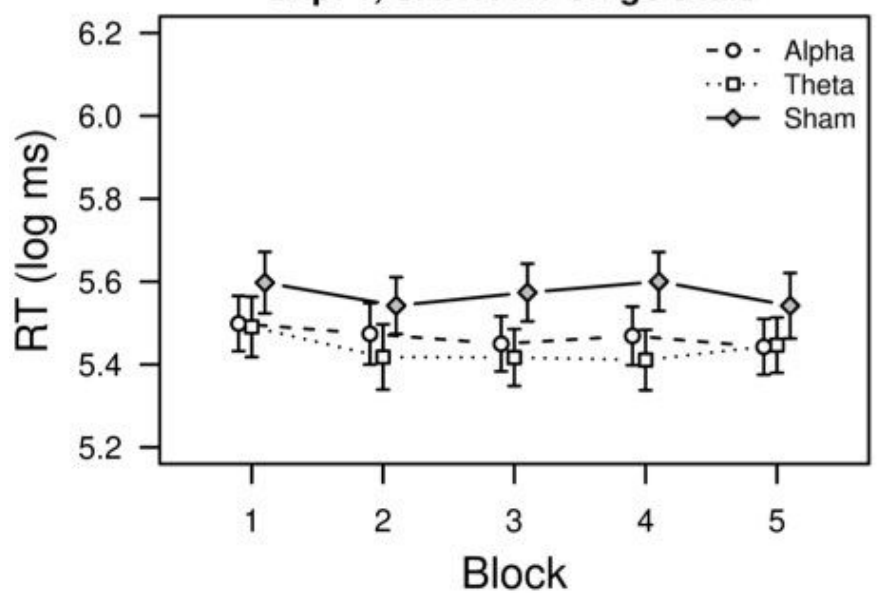

Exp. 1, SART: Accuracy on no-go trials

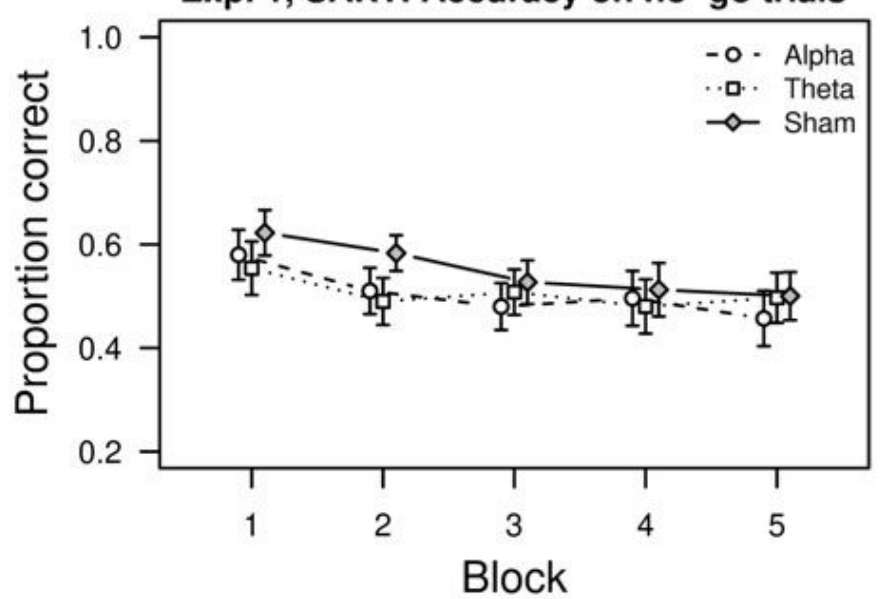

Exp. 2, PVT: RT

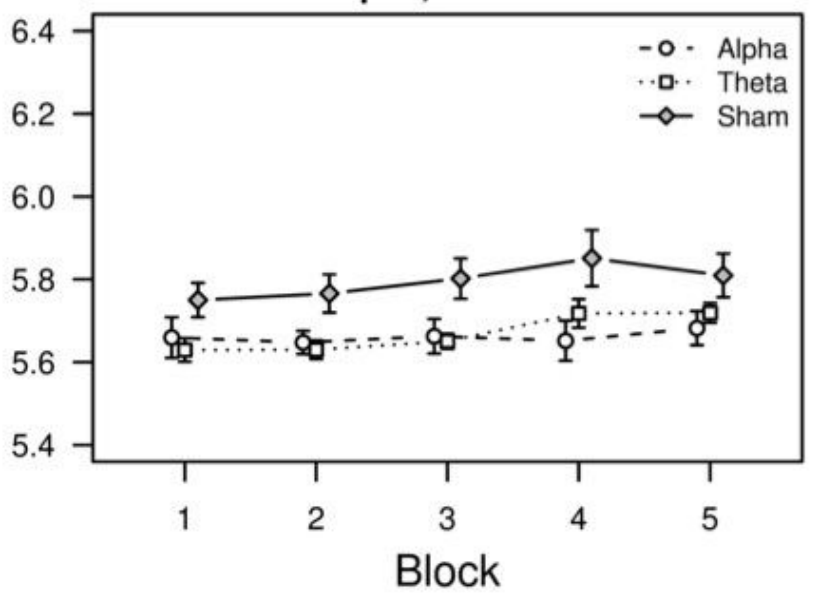

Exp. 2, SART: RT on go trials

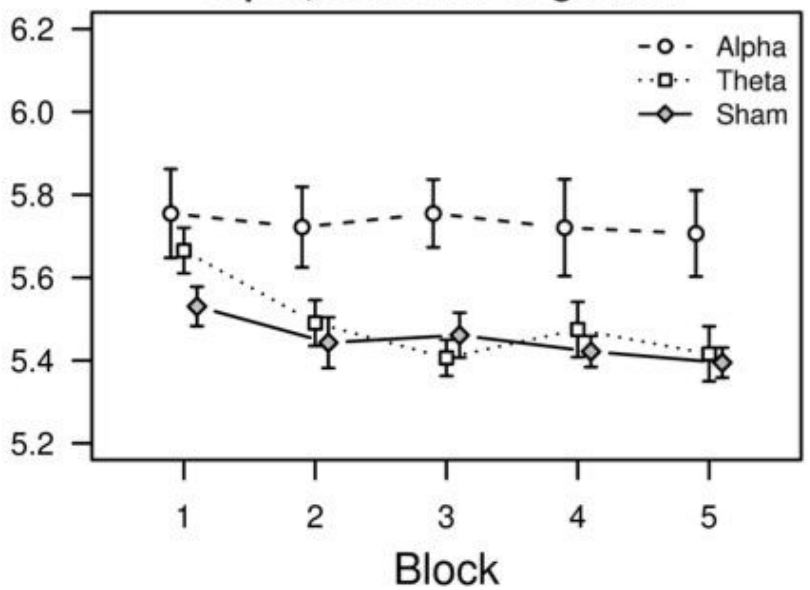

Exp. 2, SART: Accuracy on no-go trials

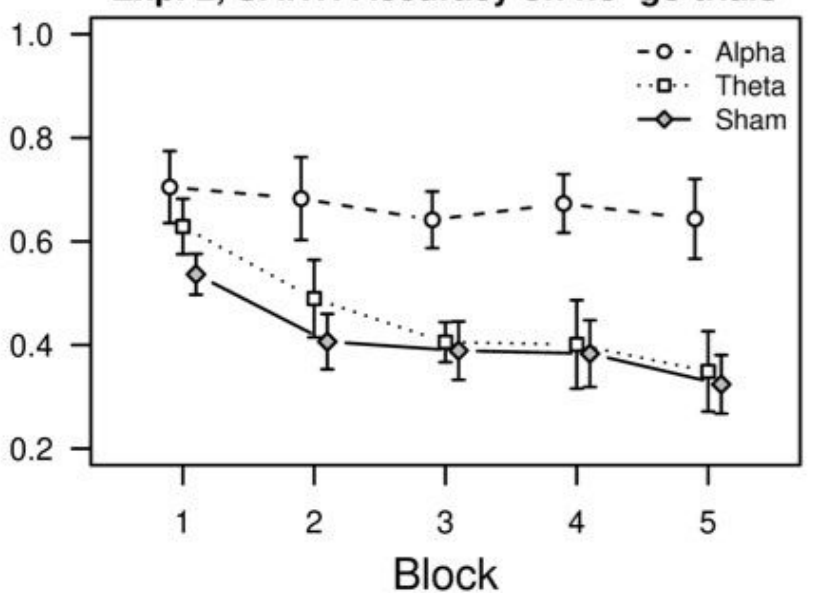

Figure 2

Arousal (PVT) and executive (SART) vigilance performance as a function of tACS condition (Alpha, Theta or Sham) across time-on-task. 